\title{
Analytic solutions of the Thomas equation by generalized tanh and travelling wave hypothesis methods
}

\author{
K. S. Al-Ghafri \\ Department of General Requirements, College of Applied Sciences - Nizwa, \\ P.O. Box: 699 Nizwa, Postal Code: 611, Oman \\ E-mail: kh22gh@gmail.com
}

\begin{abstract}
The analytical solutions of the Thomas equation are investigated. The wave transformation is exploited to simplify the Thomas equation from a form of partial differential equation (PDE) to an ordinary differential equation (ODE). Both of the generalised tanh and the travelling wave hypothesis methods are applied to obtain exact solutions for the Thomas equation.
\end{abstract}

Keywords: Thomas equation; Generalised tanh method; Travelling wave hypothesis Method; Exact solution; Solitary wave solution.

\section{Introduction}

The study of nonlinear PDEs has become the subject of theoretical attention due to its applications on many natural and physical sciences like fluid dynamics, plasma physics, ocean and atmospheric waves, mathematical biology, chemistry, etc.

In the last decade, a search of analytical exact solutions to the nonlinear equations has been the interest of many authors. Therefore, many effective methods such as Darboux transformation [5, 15], Bäcklund transformation $[13,8]$, the F-expansion method [7, 14], Exponent function method [6, 4], sine - cosine method [16, 17], Jacobi elliptic function method $[3,2]$, the tanh-function method $[9,17]$, etc, are introduced to obtain different types of exact solutions for nonlinear equations.

Recently, the improvement in the tanh method with the use of the solution ansatz of a well-known equation, like Riccati equation, has become physically natural idea to obtain new efficient solutions for nonlinear PDEs. Moreover, several types of travelling wave solution can be constructed with the aid of hyperbolic auxiliary functions.

The Thomas equation is one of the important equations in physical sciences. This equation has application in the study of chemical exchange processes [10]. There are some authors who dealt with the Thomas equation intensively. For example, [10] scoped into the Lie-Bäcklund algebra of the Thomas equation to obtain analytic solution. Furthermore, [18] studied the Thomas equation by modifying the Thomas-Rosales transformation to find more general solutions. Using auto-Bäcklund transformation which is more general than both of the WeiGao-Zhang transformation and the Thomas-Rosales transformation, [19] investigated the Thomas equation and obtained some new solutions.

In this paper, we study the Thomas equation to obtain exact solutions. This equation is solved by using the generalised tanh and the travelling wave hypothesis methods to find analytic solutions. The paper is organised as follows. In the next section we analyse the idea of methods. In Section 3 we carry out the analytical study of the problem using the proposed methods. Section 4 contains our conclusions. 


\section{Mathematical analysis}

Consider a nonlinear PDE, in two independent variables, given in the form

$$
P\left(u, u_{t}, u_{x}, u_{x x}, \cdots\right)=0,
$$

where $P$ is a polynomial in its arguments.

In order to obtain travelling wave solutions, we introduce the wave transformation

$$
u(x, t)=U(\xi), \quad \xi=x+\lambda t,
$$

where $\lambda$ is a constant. Applying Equation (2), Equation (1) reduces to an ODE

$$
P\left(U, U^{\prime}, U^{\prime \prime}, \cdots\right)=0 .
$$

where prime denotes the derivative with respect to $\xi$.

\subsection{The generalised tanh method}

The generalised tanh method (see, $[1,11])$ is experessed in the following series expansion as a solution of Equation (3):

$$
U(\xi)=\sum_{i=0}^{m} a_{i} \phi^{i}
$$

where the variable $\phi=\phi(\xi)$ satisfies the Riccati equation

$$
\phi^{\prime}=\phi^{2}+k,
$$

and the integer $m$ can be determined by balancing the highest derivative term with nonlinear terms in Equation (3). Substituting Equations (4) and (5) into Equation (3) and then equating all coefficients of $\phi^{i}$ to zero, we obtain a system of algebraic equations that determines the value of constants $a_{i}, \lambda$, and $k$.

The Riccati equation (5) has different types of solutions given by

$$
\phi(\xi)= \begin{cases}-\frac{1}{\xi}, & k=0, \\ \sqrt{k} \tan (\sqrt{k} \xi), & k>0, \\ -\sqrt{k} \cot (\sqrt{k} \xi), & k>0, \\ -\sqrt{-k} \tanh (\sqrt{-k} \xi), & k<0, \\ -\sqrt{-k} \operatorname{coth}(\sqrt{-k} \xi), & k<0 .\end{cases}
$$

\subsection{The travelling wave hypothesis method}

One of the well-known solution to the nonlinear equations is the solitary wave solution. Some of solitary wave types are called bright and dark waves and these kinds of wave solutions can be obtained by assuming the following form of solution ansatz (see, [12])

$$
U(\xi)=\sigma+\mu \tanh (\eta \xi)+\rho \sec (\eta \xi)
$$

where $\sigma, \mu, \eta$ and $\rho$ are constants which will be determined by substituting Equation (7) into Equation (3). After substituting, collect coefficients of all terms with the same order of $\operatorname{sech}(\eta \xi) \tanh (\eta \xi)$ and set each coefficients to zero. Then, we obtain a system of algebraic equations for which the solution gives the values of constants $\sigma, \mu, \eta$ and $\rho$.

In the case $\sigma=\mu=0$, the bright solitary wave solution is obtained while in the limit $\rho=0$, the solution becomes the dark solitary wave solution. The existence of all constants $\sigma, \mu$ and $\rho$ gives the characteristics of both bright and dark solitary waves. 


\section{Exact solutions of the Thomas equation}

In this section, we aim to find analytic solutions to the Thomas equation which has the form

$$
u_{x t}+\alpha u_{x}+\beta u_{t}+u_{x} u_{t}=0,
$$

where $\alpha$ and $\beta$ are arbitrary constants. Equation (8), by using the wave transformation $u(x, t)=U(\xi), \xi=x+\lambda t$, reduces to an ODE

$$
U^{\prime \prime}+\omega U^{\prime}+U^{\prime 2}=0,
$$

where

$$
\omega=\frac{\alpha}{\lambda}+\beta
$$

Now, for the sake of simplicity we let $v(\xi)=U(\xi)^{\prime}$. Consequently, Equation (9) becomes

$$
v^{\prime}+\omega v+v^{2}=0 .
$$

Therefore, the exact solution of Equation (8) is obtained by

$$
u=\int^{\xi} v \mathrm{~d} \xi_{1}+c
$$

where $c$ is arbitrary constant.

\subsection{Applying the generalised tanh method}

The generalised tanh method is applied here to obtain exact solutions to the Thomas equation (8) which is converted to Equation (10) using the wave transformation. By using the ansatz (4) and (5), and balancing the term $v^{\prime}$ with the term $v^{2}$ in Equation (10), we obtain $m=1$ and thus the solution will be in the form

$$
v(\xi)=a_{0}+a_{1} \phi(\xi) .
$$

Then, substituting Equation (12) into Equation (10), using Equation (5) and equating all coefficients of $\phi^{i}(i=$ $0,1,2)$, we obtain the system of algebraic equations

$$
\begin{array}{ll}
\text { coeff. of } \phi^{2}: & a_{1}+a_{1}^{2}=0, \\
\text { coeff. of } \phi^{1}: & \omega a_{1}+2 a_{0} a_{1}=0, \\
\text { coeff. of } \phi^{0}: & a_{1} k+a_{0} \omega+a_{0}^{2}=0 .
\end{array}
$$

The solution to this system is given as

$$
\omega= \pm 2 \sqrt{-k}, \quad a_{0}=-\frac{\omega}{2}=\mp \sqrt{-k}, \quad a_{1}=-1, \quad \lambda=\frac{\alpha}{ \pm 2 \sqrt{-k}-\beta},
$$

where $\beta \neq \pm 2 \sqrt{-k}$. Hence, the use of Equations (6), (11) and (12) gives the solutions of Equation (8) as follows

$$
\begin{aligned}
& u_{1}=\mp \sqrt{-k} \xi+\ln [\cos (\sqrt{k} \xi)]+c, \quad k>0, \\
& u_{2}=\mp \sqrt{-k} \xi+\ln [\sin (\sqrt{k} \xi)]+c, \quad k>0, \\
& u_{3}=\mp \sqrt{-k} \xi+\ln [\cosh (\sqrt{-k} \xi)]+c, \quad k<0, \\
& u_{4}=\mp \sqrt{-k} \xi+\ln [\sinh (\sqrt{-k} \xi)]+c, \quad k<0,
\end{aligned}
$$

where $\xi=x+\lambda t=x+\frac{\alpha}{ \pm 2 \sqrt{-k}-\beta} t, c$ is arbitrary constant. 


\subsection{Applying the travelling wave hypothesis method}

Now, the solution of the Thomas equation is obtained by exploiting the solution ansatz (7). Applying the ansatz (7) for Equation (10) and taking the coefficients of $\operatorname{sech}^{n}(\eta \xi) \tanh ^{m}(\eta \xi)$ where $n=0,1,2$ and $m=0,1$ and then equating coefficients to zero, we obtain the following equations:

$$
\begin{array}{ll}
\text { coeff. of } \operatorname{sech}^{2}(\eta \xi): & \mu \eta-\mu^{2}+\rho^{2}=0, \\
\text { coeff. of } \tanh (\eta \xi) \operatorname{sech}(\eta \xi): & -\rho \eta+2 \mu \rho=0, \\
\text { coeff. of } \operatorname{sech}(\eta \xi): & \omega \rho+2 \sigma \rho=0, \\
\text { coeff. of } \tanh (\eta \xi): & \omega \mu+2 \sigma \mu=0, \\
\text { coeff. of } \tanh ^{0}(\eta \xi) \operatorname{sech}^{0}(\eta \xi): & \omega \sigma+\sigma^{2}+\mu^{2}=0,
\end{array}
$$

for which the solutions are

$$
\begin{aligned}
& \text { 1. } \rho=0, \quad \mu= \pm \sigma, \quad \eta= \pm \sigma, \quad \omega=-2 \sigma, \\
& \text { 2. } \rho= \pm i \sigma, \quad \mu=\mp \sigma, \quad, \eta=\mp 2 \sigma, \quad \omega=-2 \sigma .
\end{aligned}
$$

Hence, the solutions of Equation (8) are given as follows. From Equations (7), (11) and (16), we obtain the solution

$$
u_{1}=\sigma \xi+\ln (\cosh ( \pm \sigma \xi))+c .
$$

Then, Equations (7), (11) and (17) yield the solution

$$
u_{2}=\sigma \xi+\ln (\sqrt{\cosh (2 \sigma \xi)}) \mp \frac{i}{2} \arctan (\sinh (2 \sigma \xi))+c .
$$

In Equations (18) and (19), $\xi=x+\lambda t=x+\frac{\alpha}{-2 \sigma-\beta} t, \beta \neq-2 \sigma, i=\sqrt{-1}, \sigma$ and $c$ are arbitrary constants.

In what follows, we display some non-complex analytical solutions obtained by the generalised tanh method to give more insight into the behaviour of waves. We take the solutions $u_{3}$ and $u_{4}$ with the positive value of the first term in both of them.
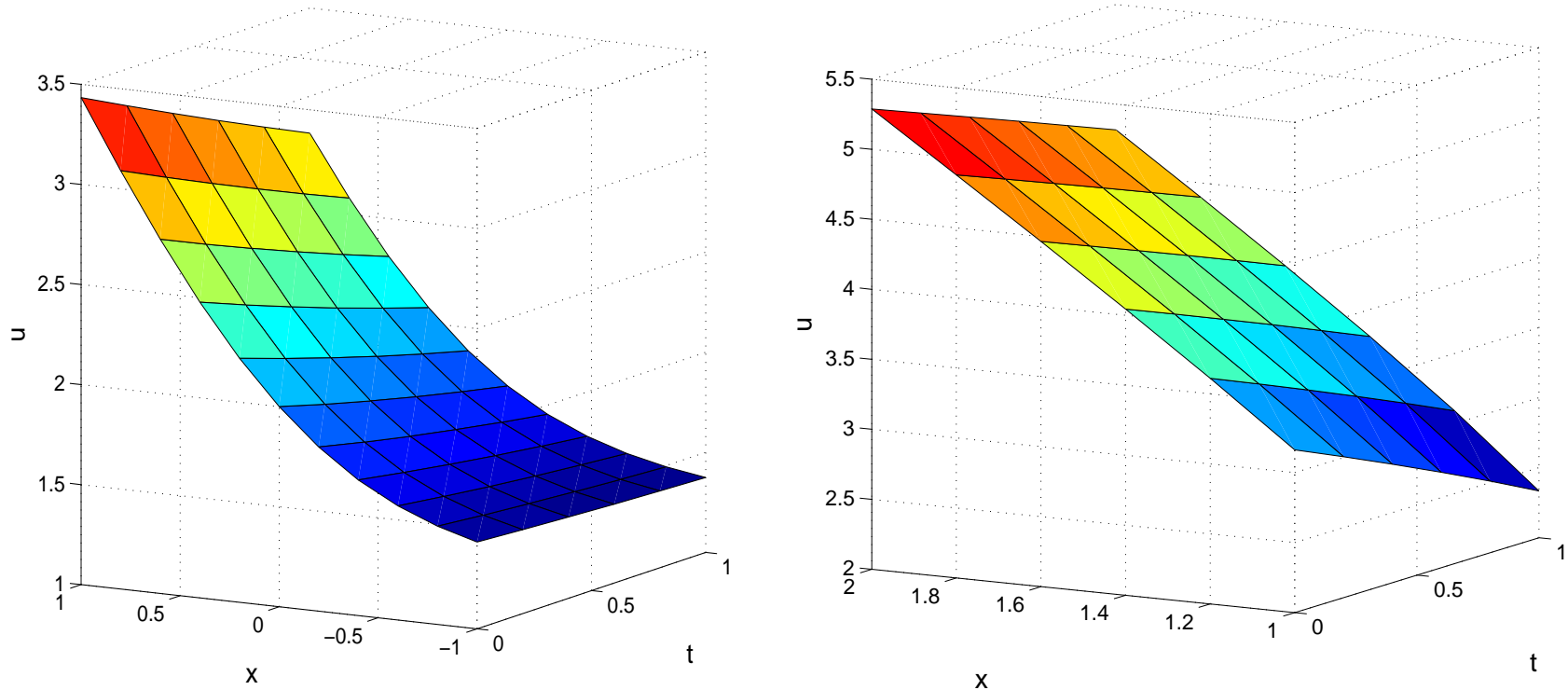

Figure 1: Left: The solution $u_{3}$. Right: The solution $u_{4}$. In both cases: $k=-1, \alpha=1, \beta=1$ and $c=2$.

\section{Conclusion}

In this article, the generalised tanh and the travelling wave hypothesis methods were employed in order to find the exact solutions of the Thomas equation. There were different types of solutions that obtained by proposed methods, where some of them were complex solutions. The used methods are powerful and applicable to a wide range of nonlinear equations. 


\section{References}

[1] E. Fan and Y. C. Hon. Generalized tanh method extended to special types of nonlinear equations, Z. Naturforsch, 57a, (2002) 692-700.

[2] E. Fan and J. Zhang. Applications of the jacobi elliptic function method to special-type nonlinear equations, Physics Letters A, 305, (2002) 383-392.

[3] Z. T. Fu, S. K. Liu, S. D. Liu, and Q. Zhao. New jacobi elliptic function expansion and new periodic solutions of nonlinear wave equations, Physics Letters A, 290, (2001) 72-76.

[4] D. D. Ganji, A. Asgari, and Z. Z. Ganji. Exp-function based solution of nonlinear Radhakrishnan, Kundu and Laskshmanan (RKL) equation, Acta Appl Math, 104, (2008) 201-209.

[5] X. G. Geng and H. W. Tam. Darboux transformation and soliton solutions for generalised nonlinear Schrödinger equations, Journal of the Physical Society of Japan, 68, (1999) 1508-1512.

[6] J. H. He and M. A. Abdou. New periodic solutions for nonlinear evolution equations using Exp-function method, Chaos Solitons Fractals, 34, (2007) 1421-1429.

[7] J. B. Liu and K. Q. Yang. The extended F-expansion method and exact solutions of nonlinear PDEs, Chaos Solitons Fractals, 22, (2004) 111-121.

[8] L Luo. New exact solutions and Bäcklund transformation for Boiti-Leon-Manna-Pempinelli equation, Physics Letters A, 375, (2011) 1059-1063.

[9] W. Malfliet and W. Hereman. The tanh-method part I. Exact solutions of nonlinear evolution and wave equations, Phys. Script., 54, (1996) 563-568.

[10] S. Y. Sakovich. On the Thomas equation, J. Phys. A: Math. Gen., 21, (1988) L1123-L1126.

[11] N. Taghizadeh. Generalized tanh method with the Riccati equation for solving the Sixth-Order Ramani equation, Mathematica Aeterna, 2, (2012) 483-487.

[12] H. Triki and A. Biswas. Complexition and solitary wave solutions of the $(2+1)$-dimensional dispersive long wave equations, CJMS, 1, (2012) 39-46.

[13] H. D. Wahlquist and F. B. Estabrook. Bäcklund transformation for solutions of the Korteweg-de Vries equation, Phys. Rev. Lett., 31, (1973) 1386-1390.

[14] J. Wang. Construction of new exact traveling wave solutions to (2+1)-dimensional mVN equation, International Journal of Nonlinear Science, 9, (2010) 325-329.

[15] Y. Wang, L. J. Shen, and D. L. Du. Darboux transformation and explicit solutions for some (2+1)-dimensional equations, Physics Letters A, 366, (2007) 230-240.

[16] A. M. Wazwaz. A sine-cosine method for handling nonlinear wave equations, Math. Comput. Model., 40, (2004) $499-508$.

[17] A. M. Wazwaz. The tanh and the sine-cosine methods for compact and noncompact solutions of the nonlinear KleinGordon equation, Appl. Math. Comput., 167, (2005) 1179-1195.

[18] G. M. Wei, Y. T. Gao, and H. Zhang. On the Thomas equation for the ion-exchange operations, Czech. J. Phys., 52, (2002) 749-751.

[19] Z. Yan. Study of the Thomas equation: a more general transformation (auto-Backlund transformation) and exact solutions, Czech. J. Phys., 53, (2003) 297-300. 